\title{
Molecular Dynamics Simulation of Structural Characterization of Elastic and Inelastic Deformation in $\mathrm{ZrCu}$ Metallic Glasses
}

\author{
Shidong Feng, ${ }^{1}$ Li Qi, ${ }^{1}$ Gong Li, ${ }^{1,2}$ and Riping Liu' \\ ${ }^{1}$ State Key Laboratory of Metastable Materials Science and Technology, Yanshan University, Qinhuangdao 066004, China \\ ${ }^{2}$ Department of Materials Science and Engineering, The University of Tennessee, Knoxville, TN 37996, USA
}

Correspondence should be addressed to Li Qi; qili@ysu.edu.cn and Riping Liu; riping@ysu.edu.cn

Received 4 March 2014; Accepted 10 May 2014; Published 27 May 2014

Academic Editor: Alireza Khataee

Copyright (C) 2014 Shidong Feng et al. This is an open access article distributed under the Creative Commons Attribution License, which permits unrestricted use, distribution, and reproduction in any medium, provided the original work is properly cited.

\begin{abstract}
The nanoscopic deformation behaviors in a $\mathrm{ZrCu}$ metallic glass model during loading-unloading process under uniaxial compression have been analyzed on the basis of the molecular dynamics (MD). The reversible degree of shear origin zones (SOZs) is used as the structural indicator to distinguish the elastic deformation and inelastic deformation of $\mathrm{ZrCu}$ metallic glass at the atomic level. We find that the formation of SOZs is reversible at the elastic stage but irreversible at the inelastic stage during the loading and unloading processes. At the inelastic stage, the full-icosahedra fraction in SOZs is quickly reduced with increased strain and the decreasing process is also irreversible during the unloading processes.
\end{abstract}

\section{Introduction}

Bulk metallic glasses (BMGs) have attracted much attention as novel potential engineering materials because of their specific mechanical properties [1-3]. However, compared with their crystalline counterparts, many extraordinary behaviors in glasses are still poorly understood [4-6]. One of the most well-known features is the very high elastic strain limit of BMGs, which is about one order of magnitude higher than that in conventional crystalline metals and alloys [79]. However, it is known that the global plasticity of BMGs is very low $[10,11]$, which is caused by avalanche of shear transformation zones (STZs). The shear transformation zone (STZ) was hypothesized as the small cluster containing tens of atoms confined within elastic medium [11]. High-purity metallic-glass materials only show the elastic deformation or very limited plastic deformation under unconstrained conditions [12]. It is of great significance to understand the microcosmic differences between elastic strain and plastic strain. Therefore, studying structural characterization of elastic and inelastic deformation in BMGs at the atomic level is particularly important.

Recently, some researchers provide some details on the elastic deformation of amorphous solids [13-16]. For instance, Hufnagel reports the use of high-energy X-ray scattering to measure strain in a $\mathrm{Zr}_{57} \mathrm{Ti}_{5} \mathrm{Cu}_{20} \mathrm{Ni}_{8} \mathrm{Al}_{10}$ bulk metallic glass in situ during uniaxial compression in the elastic regime [15]. In addition, much work has been devoted to the inhomogeneous plastic responses (shear transformation events) in MGs [17-21]. However, the exact correlation of the degree of elastic and inelastic deformation with the internal structure in MGs at the atomic level is still unresolved. This condition can be attributed to the very limited existing experimental method for detecting atomic motion. Considering the experimental difficulty of directly measuring the movement of an atom or determining which local structures are prone to form, we have focused on computer simulation of simple alloy systems. This technique eliminates some of the problems encountered in experiments and studies the deformation processes at the atomic level.

Given the lack of a structural indicator for making a distinction between elastic and inelastic deformation, understanding the microscopic deformation mechanism in MGs on the whole is difficult [22]. In this letter, the nanoscopic deformation behaviors in a $\mathrm{ZrCu}$ metallic-glass model during loading-unloading process under uniaxial compression have been analyzed on the basis of the molecular dynamics (MD). And we employ the reversible degree of shear origin zones (SOZs) as structural indicator to distinguish the elastic and inelastic deformation processes in MGs. 


\section{Theoretical Model and Calculation Details}

2.1. Preparation of $\mathrm{ZrCu} M G$. In our molecular dynamics (MD) simulations, the model system of $\mathrm{ZrCu} \mathrm{MG}$ was supplied in a large-scale atomic/molecular massively parallel simulator (LAMMPS) [23]. The force field used in this work is a realistic embedded atom method (EAM) potential [24], which is suitable for computing pairwise interactions for metals and metal alloys. The dimensions of the model structures used in the calculations are $17.1 \mathrm{~nm} \times 25.6 \mathrm{~nm} \times$ $4.3 \mathrm{~nm}$ in the $x$-, $y$-, and $z$-directions, respectively. The structures contained approximately 100,000 atoms. The model was heated from $300 \mathrm{~K}$ to $2500 \mathrm{~K}$ at a constant rate of $10 \mathrm{~K} \mathrm{ps}^{-1}$. To make the state of the system as natural as possible, we relaxed the liquid system for 50 ps at $2500 \mathrm{~K}$ within the NPT ensemble (namely, constant number, constant pressure, and constant temperature, and the temperature was controlled by Nose-Hoover thermostat method) under periodic boundary conditions. Next, we cooled the system from $2500 \mathrm{~K}$ down to $100 \mathrm{~K}$ in $100 \mathrm{~K}$ decrements at a constant quenching rate of $5 \mathrm{~K} \mathrm{ps}^{-1}$ at zero external pressure in the NPT ensemble.

2.2. Compression of $\mathrm{ZrCu} M G$. Afterwards, compression at a constant strain rate of $1 \times 10^{8} \mathrm{~s}^{-1}$ along the $y$-direction was loaded at a constantly low temperature of $50 \mathrm{~K}$. Periodic boundary conditions were imposed in the $y$-and $z$-directions and free surfaces in the $x$-direction to allow the occurrence of shear offset on the free surfaces. To simulate plane strain state, we maintained the pressure of the $z$-direction at zero.

\section{Results and Discussion}

3.1. The Compressive Stress-Strain Curve of $\mathrm{ZrCu} \mathrm{MG.} \mathrm{The}$ compressive stress-strain curve of $\mathrm{ZrCu} \mathrm{MG}$ is shown in Figure 1. The yield stress is linear when the strain is $<4 \%$, suggesting an elastic deformation stage. The simulation result is in good agreement with the result obtained by Cao [25]. With increased strain, the rise of stress values becomes slow, showing a platform corresponding to the inelastic deformation. The stress reaches a maximum of $2.3 \mathrm{GPa}$ at a strain of about $6 \%$. To clarify the microstructure evolution at different stages of the system, $2 \%$ and $5 \%$ compressive strain of the system at the imposed $y$-direction, respectively, are analyzed.

3.2. Change in $x$-Displacement under Relaxation Process of Elastic and Inelastic Deformation. To display the relaxation process of elastic and inelastic deformation, we used the degree of $x$-displacement of each atom as the structural indicator, as shown in Figure 2. It is important to note that there are also $y$-displacement and $z$-displacement, and we just put the $x$-displacement as a representative to characterize the relaxation process. Figures $2(\mathrm{a})-2(\mathrm{~d})$ show the relaxation process by monitoring $x$-displacements after unloading $2 \%$ stress. Figures $2\left(a^{\prime}\right)-2\left(d^{\prime}\right)$ reveal the relaxation process under 5\% strain. In Figure 2(a), some $x$-displacements exist after compressing the $\mathrm{ZrCu}$ system. However, after $150 \mathrm{ps}$ relaxation (Figures 2(b)-2(d)), $x$-displacements gradually

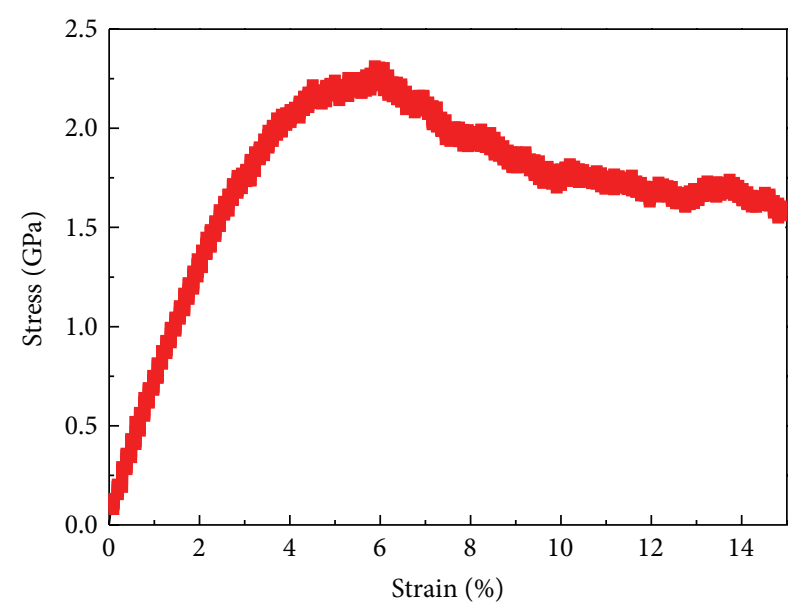

FIgUre 1: Typical stress-strain curve of $\mathrm{ZrCu}$ MG as a function of overall sample strain.

disappear, suggesting that the $x$-displacements of each atom are reversible at the elastic deformation stage. On the other hand, the $x$-displacements of the system with $5 \%$ strain are strongly evident, as shown in Figure $2\left(\mathrm{a}^{\prime}\right)$. After 150 ps relaxation, the $x$-displacements gradually recover but numerous $x$-displacements still exist in the system, as shown in Figure $2\left(\mathrm{~d}^{\prime}\right)$. Figures $2\left(\mathrm{a}^{\prime}\right)-2\left(\mathrm{~d}^{\prime}\right)$ suggest that these $x$ displacements are irreversible at a strain of $5 \%$, which correspond to the inelastic deformation stage. This observation also indicates that some atoms cannot go back to the initial location at the inelastic deformation stage. The author Zhang et al. also reported that the instantaneous elastic strain is observed to vanish immediately as the applied stress is removed (unloading process), while inelastic strain that decays beyond the simulation timescale is still remained [26]. This validates our results and implies that, with the increase in strain, some atoms deviate from their original positions, causing the local structure to be destroyed. Even after a long period of relaxation, these atoms cannot be restored to their original locations. To eliminate system size effects, we also structured a bigger model whose dimensions were $27.5 \mathrm{~nm} \times$ $5.5 \mathrm{~nm} \times 55 \mathrm{~nm}$ in the $x$-, $y$-, and $z$-directions, respectively, containing approximately 500,000 atoms. And we found that the main results presented above do not change.

\subsection{Change in $\eta_{i}^{\text {Misses }}$ under Relaxation Process of Elastic and} Inelastic Deformation. To further explain the phenomenon of the $x$-displacement in the relaxation process, we use the atomic local shear strain $\eta_{i}^{\text {Misses }}$ as a structural indicator to monitor deformation processes [27]. We use relaxed glass prior to performing uniaxial compression deformation in the $y$-direction as a reference. Atoms in Figure 3 are colored according to their atomic shear strain $\eta_{i}^{\text {Misses }}$. Regions that have undergone larger shear strain (light blue) have a relatively large $\eta_{i}^{\text {Misses }}$.

Shear origin zones (SOZs) are the regions in a metallic glass where strain becomes inhomogeneous [28]. A typical experimentally observed shear band width in a metallic glass 


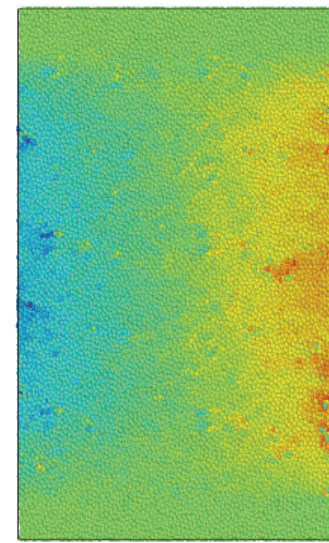

(a)

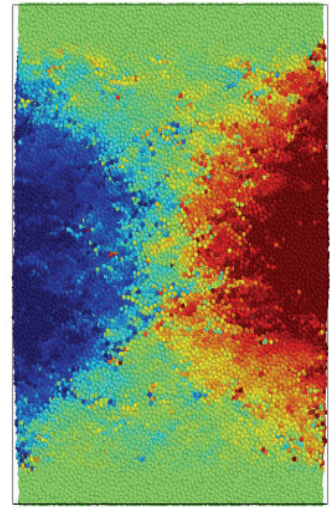

$\left(a^{\prime}\right)$

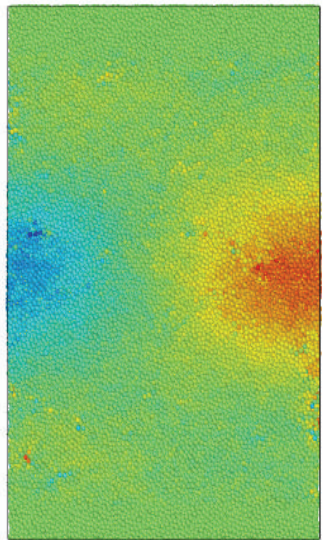

(b)

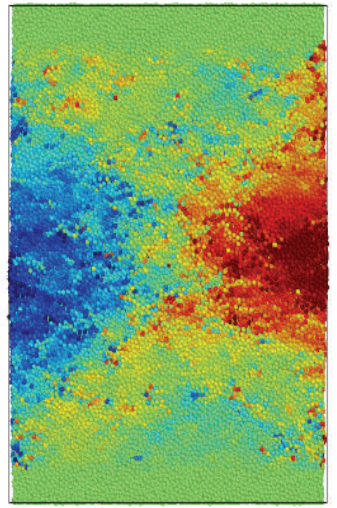

$\left(b^{\prime}\right)$

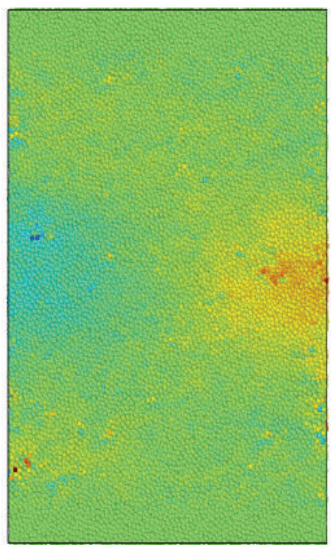

(c)

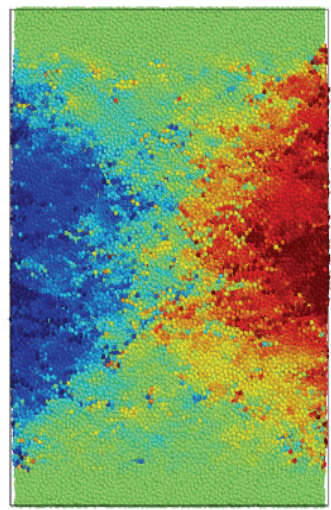

$\left(c^{\prime}\right)$

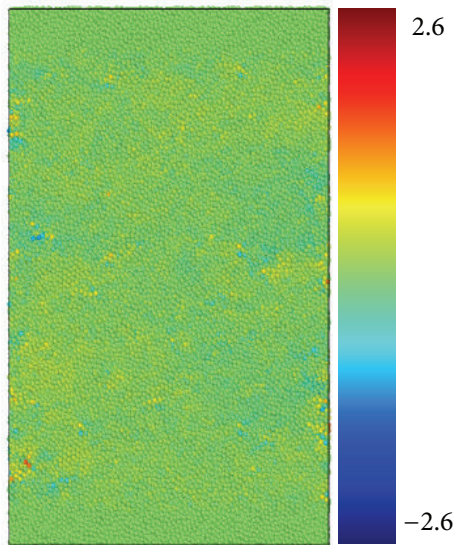

(d)

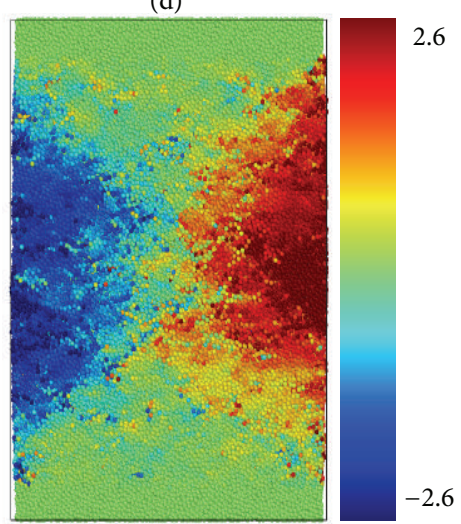

$\left(d^{\prime}\right)$

FIGURE 2: Relaxation process observed by monitoring $x$-displacement after unloading the stress of $\mathrm{ZrCu}$ MG. (a)-(d) compressed by $2 \%$ strain. $\left(\mathrm{a}^{\prime}\right)-\left(\mathrm{d}^{\prime}\right)$ compressed by $5 \%$ strain. The red region represents the atoms moving along the positive $x$-direction, and the blue region represents the atoms moving along the negative $x$-direction.

is on the order of $10 \mathrm{~nm}$ to $20 \mathrm{~nm}$ [29], but the size of SOZs is about $1 \mathrm{~nm}$ to $2 \mathrm{~nm}$. Therefore, SOZs are smaller than shear bands. In Figure 3(a), some SOZs can be clearly observed after $2 \%$ strain compression of the $\mathrm{ZrCu}$ systems. However, after 150 ps relaxation (Figure 3(d)), fewer SOZs are observed, suggesting that the formation of SOZs is reversible at the elastic deformation stage. In Figure 3( $\left.\mathrm{a}^{\prime}\right)$, many SOZs can be observed after 5\% strain compression of the $\mathrm{ZrCu}$ systems. After 150 ps relaxation (Figure $3\left(\mathrm{~d}^{\prime}\right)$ ), many SOZs are still observed, suggesting that the formation of SOZs is irreversible at the inelastic deformation stage. The quantity and size of the SOZs in Figure $3\left(\mathrm{a}^{\prime}\right)$ are larger than those of the SOZs in Figure 3(a). This observation indicates that new SOZs form with increased strain and the origins increase in size. With increased strain, these SOZs interact with one another and develop into shear bands. Smaller amounts of SOZs can be seen in Figure 3(d), whereas numerous SOZs can be observed in Figure $3\left(\mathrm{~d}^{\prime}\right)$. This observation implies that, with increased SOZ size to a certain value, the formation of SOZs becomes irreversible. Hence, we propose that elastic deformation is related to these SOZs in MGs. The degree of SOZs in MGs can be controlled, and the plasticity of metallic glass can be improved [28].
3.4. Change in the Fraction of FI in SOZs under Relaxation Process. To further explain the structural features of SOZs at different deformation stages of $\mathrm{ZrCu}$ metallic glass, the Voronoi tessellation method was used [30]. In this method, the simulation cell is divided into Voronoi polyhedra (VPs) around each atom. A VP is defined as the minimal polyhedron whose planar faces bisect at right angles the lines joining an atom to its neighboring atoms. The polyhedra can be characterized by the Voronoi index $\left[n_{3}, n_{4}, n_{5}, n_{6}\right]$, where $n_{i}$ denotes the number of $i$-edged faces of the VP. An $i$-edged face reflects the local symmetry of the central atom with some nearest neighbor atoms in a certain direction.

In previous studies, the $\mathrm{Cu}$-centered Voronoi polyhedron with index $[0,0,12,0]$ (full icosahedron (FI)) has been found to be a key structural motif in amorphous $\mathrm{Cu}-\mathrm{Zr}$ alloys [31]. FI units with high packing density and high shear resistance reportedly determine the plasticity of $\mathrm{Cu}$-Zr glasses.

For our model $\mathrm{ZrCu} \mathrm{MG}$, we observed a clear difference between the responses of $\mathrm{Cu}$ atoms in $\mathrm{Cu}$-centered full icosahedra [Cu-FI; Voronoi index $(0,0,12,0)$ clusters)] and those that do not have full-icosahedra surroundings (Cu-non-FI). The Voronoi tessellation analysis shows that $21.5 \%$ of $\mathrm{Cu}$ atoms are centers of full icosahedra in this sample. In our Voronoi analysis, the cutoff is chosen large 


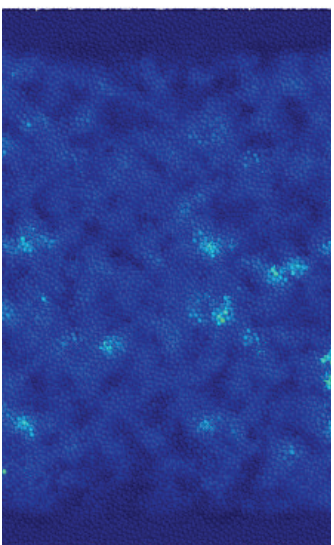

(a)

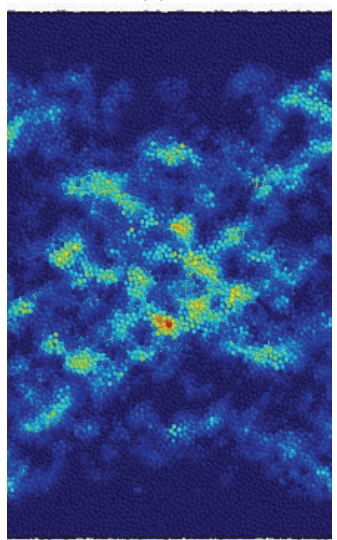

$\left(a^{\prime}\right)$

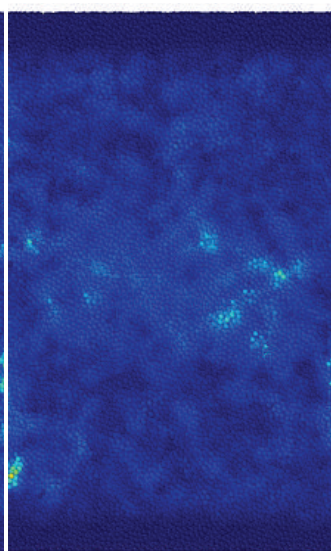

(b)

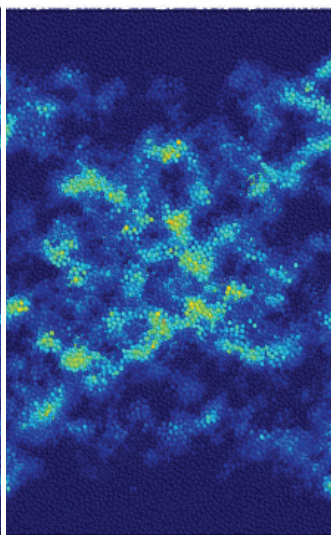

$\left(b^{\prime}\right)$

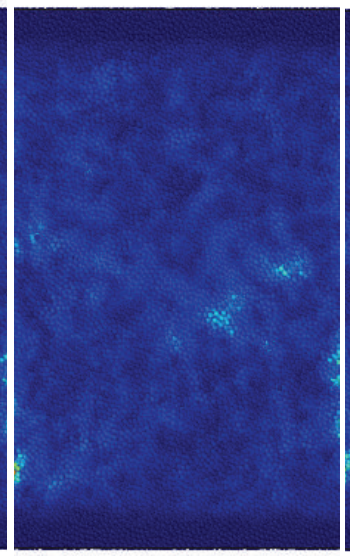

(c)

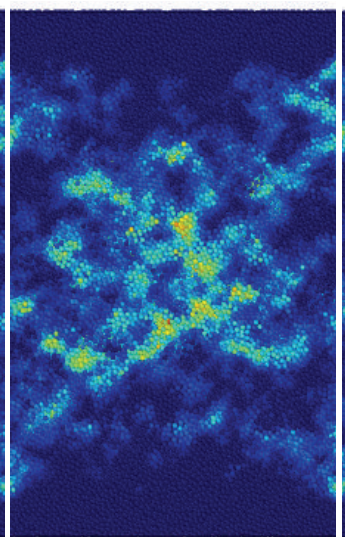

$\left(c^{\prime}\right)$

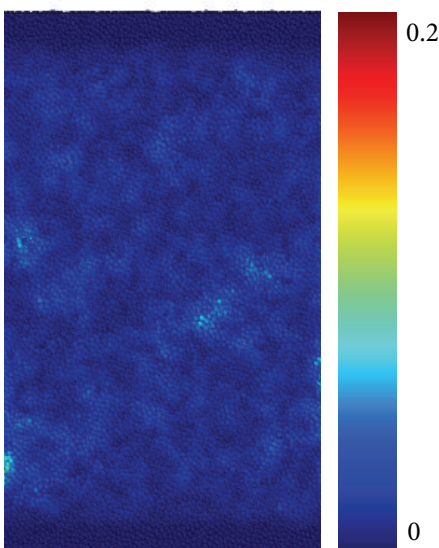

(d)

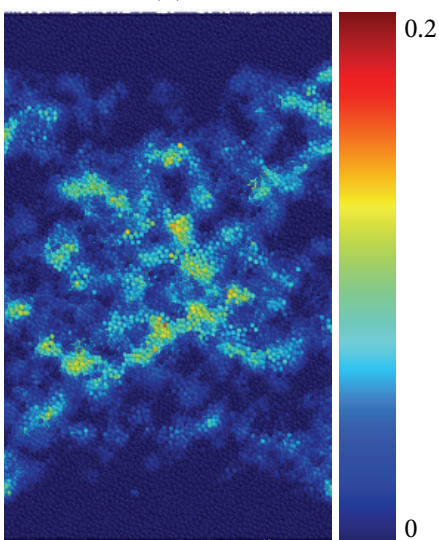

$\left(d^{\prime}\right)$

FIGURE 3: Relaxation process observed by monitoring $\eta_{i}^{\text {Misses }}$ after unloading the stress of ZrCu MG. (a)-(d) compressed by $2 \%$ strain. $\left(\mathrm{a}^{\prime}\right)-\left(\mathrm{d}^{\prime}\right)$ compressed by $5 \%$ strain. Regions that have undergone larger shear strain (light blue) have relative large $\eta_{i}^{\text {Misses }}$.

enough (i.e., $5 \AA$ ) so that the Voronoi indices distributions are converged. Meanwhile, we also performed the analyses using a cutoff of $3.8 \AA$, which corresponds to the first minimum of $g(r)$, and found that the main results do not change.

Figure 4(a) shows the fraction of $\mathrm{Cu}$-centered full icosahedra in SOZs during deformation before the strain reaches $\sim 5 \%$. Evidently, the fraction of $\mathrm{Cu}$-centered full icosahedra is maintained at $21 \%$ before the strain reaches $4 \%$. Lekka et al. reported that the numbers of icosahedral clusters (ICO) destroyed and created within the elastic regime are almost the same, resulting in a dynamically constant number of ICO clusters [32]. However, a sudden and rapid drop in the fraction of $\mathrm{Cu}$-centered full icosahedra is observed at $\varepsilon=4 \%$, corresponding to the nonlinear section of the stressstrain curve (Figure 1). This observation indicates that elastic deformation translates to inelastic deformation associated with a drop of the numbers of the full icosahedra in SOZs. Meanwhile, along with the drop of the numbers of the full icosahedra in SOZs, the efficient packing for this composition is destroyed to some degree and the energy of these zones increases. Hence, the stability of SOZs is weakened and the structures become inhomogeneous.
To check whether the drop of the numbers of the $\mathrm{Cu}$ centered full icosahedra can recover to the initial fraction, the $\mathrm{ZrCu}$ systems compressed by $2 \%$ and $5 \%$ strain were analyzed, as shown in Figure 4(b). With increased relaxation time, the fraction of $\mathrm{Cu}$-centered polyhedra of the $\mathrm{ZrCu}$ systems compressed by $5 \%$ returns to $\sim 19.3 \%$ from $\sim 18.33 \%$, whereas the fraction of $\mathrm{Cu}$-centered polyhedra of the $\mathrm{ZrCu}$ systems compressed by $2 \%$ returns to $\sim 21.5 \%$ from $\sim 21.1 \%$. The fraction of $\mathrm{Cu}$-centered polyhedra of the $\mathrm{ZrCu}$ systems before being compressed is $\sim 21.5 \%$, as shown by the line of $0 \%$ strain in Figure 4(b). This fraction testifies the deformation along with decreasing the fractions of $\mathrm{Cu}$-centered full icosahedra. Before $\varepsilon=4 \%$, the decreasing process is reversible; after $\varepsilon=4 \%$, the decreasing process becomes irreversible. This finding indicates that FI in SOZs is partially destroyed at the inelastic deformation stage. Interestingly, both fractions of $\mathrm{Cu}$-centered polyhedra of the $\mathrm{ZrCu}$ systems compressed by $2 \%$ strain and $5 \%$ strain increase at the initial stage of relaxation. This phenomenon is facilitated by the additional energy introduced by the large stress overshoot caused by the high simulated strain rate. Therefore, the phenomenon leads to a recovery of the atomic structure, that is, a decrease in free volume and an increase in FI density. 


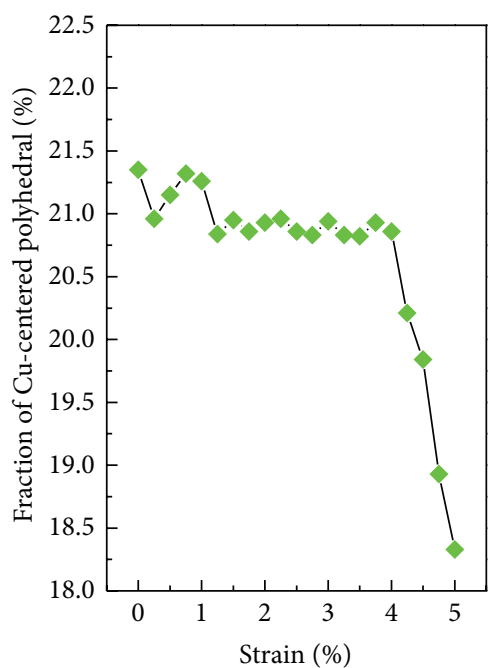

(a)

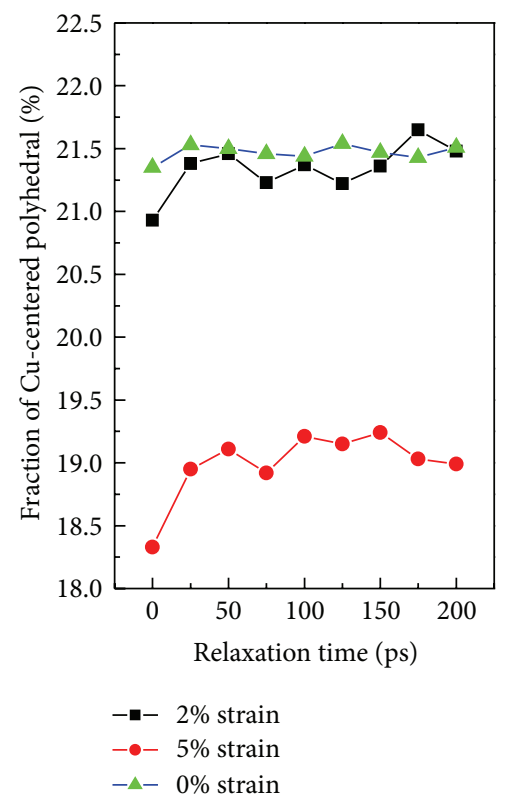

(b)

Figure 4: (a) Fraction of $\mathrm{Cu}$-centered full icosahedra at different strains during compression deformation. (b) Fractions of Cu-centered full icosahedra as a function of relaxation time. To show whether the drop of the numbers of the Cu-centered full icosahedra can recover to the initial fraction, relaxation of the sample compressed by $0 \%, 2 \%$, and $5 \%$ compressive strain, respectively, is analyzed.

\section{Conclusion}

In conclusion, elastic deformation is related to the reversible degree of SOZs. We find that the formation of SOZs is reversible at the elastic stage but is irreversible at the inelastic stage. At the elastic deformation stage, the fraction of FI in SOZs remains at a high grade. However, the fraction of FI in SOZs quickly decreases with increased strain at the inelastic deformation stage, and the decreasing process is also irreversible. This finding implies that the FI structure is destroyed and that the local structural environments of the SOZs are changed. Therefore, we can change the degree of SOZ localization to adjust plastic deformation and accordingly provide foundations for basic research on and future applications of MG.

\section{Conflict of Interests}

The authors declare that there is no conflict of interests regarding the publication of this paper.

\section{Acknowledgments}

The authors thank Shaopeng Pan from Peking University for helpful discussion. This work was supported by the National Basic Research Program of China (2013CB733000) and the National Natural Science Foundation of China (51271161 and 51271162).

\section{References}

[1] C. A. Pampillo, "Flow and fracture in amorphous alloys," Journal of Materials Science, vol. 10, no. 7, pp. 1194-1227, 1975.

[2] H. S. Chen and D. E. Polk, "Mechanical properties of NiFe based alloy glasses," Journal of Non-Crystalline Solids, vol. 15, no. 2, pp. 174-178, 1974.

[3] K. Kondoh, K. Kawabata, T. Serikawa, and H. Kimura, "Structural characteristics and crystallization of metallic glass sputtered films by using $\mathrm{Zr}$ system target," Advances in Materials Science and Engineering, vol. 2008, Article ID 312057, 4 pages, 2008.

[4] D. Srolovitz, V. Vitek, and T. Egami, "An atomistic study of deformation of amorphous metals," Acta Metallurgica, vol. 31, no. 2, pp. 335-352, 1983.

[5] J. Lu, G. Ravichandran, and W. L. Johnson, "Deformation behavior of the $\mathrm{Zr}_{41.2} \mathrm{Ti}_{13.8} \mathrm{Cu}_{12.5} \mathrm{Ni}_{10} \mathrm{Be}_{22.5}$ bulk metallic glass over a wide range of strain-rates and temperatures," Acta Materialia, vol. 51, no. 12, pp. 3429-3443, 2003.

[6] C. D. Lorenz and M. J. Stevens, "Fracture behavior of LennardJones glasses," Physical Review E-Statistical, Nonlinear, and Soft Matter Physics, vol. 68, no. 2, Article ID 021802, 6 pages, 2003.

[7] C. A. Schuh, T. C. Hufnagel, and U. Ramamurty, "Mechanical behavior of amorphous alloys," Acta Materialia, vol. 55, no. 12, pp. 4067-4109, 2007.

[8] W. H. Wang, "The elastic properties, elastic models and elastic perspectives of metallic glasses," Progress in Materials Science, vol. 57, no. 3, pp. 487-656, 2012.

[9] G. Li, Y. Y. Wang, P. K. Liaw, Y. C. Li, and R. P. Liu, "Electronic structure inheritance and pressure-induced polyamorphism in lanthanide-based metallic glasses," Physical Review Letters, vol. 109, no. 12, Article ID 125501, 2012. 
[10] W. L. Johnson and K. Samwer, "A universal criterion for plastic yielding of metallic glasses with a $(\mathrm{T} / \mathrm{Tg})^{2 / 3}$ temperature dependence," Physical Review Letters, vol. 95, no. 19, Article ID 195501, 2005.

[11] M. Chen, "Mechanical behavior of metallic glasses: microscopic understanding of strength and ductility," Annual Review of Materials Research, vol. 38, pp. 445-469, 2008.

[12] D. Qiao, L. Huang, G. Wang, and P. K. Liaw, "Strain rate effects on the deformation behavior of $\left(\mathrm{Zr}_{55} \mathrm{Al}_{10} \mathrm{Ni}_{5} \mathrm{Cu}_{30}\right)_{99} \mathrm{Y}_{1}$ and $\mathrm{Cu}_{45} \mathrm{Zr}_{47.5} \mathrm{Ag}_{7.5}$ bulk metallic glasses," Materials Science and Engineering A, vol. 526, no. 1-2, pp. 69-73, 2009.

[13] M. Tsamados, A. Tanguy, C. Goldenberg, and J.-L. Barrat, "Local elasticity map and plasticity in a model Lennard-Jones glass," Physical Review E-Statistical, Nonlinear, and Soft Matter Physics, vol. 80, no. 2, Article ID 026112, 17 pages, 2009.

[14] M. J. Demkowicz and A. S. Argon, "Autocatalytic avalanches of unit inelastic shearing events are the mechanism of plastic deformation in amorphous silicon," Physical Review BCondensed Matter and Materials Physics, vol. 72, no. 24, Article ID 245206, 2005.

[15] T. C. Hufnagel, R. T. Ott, and J. Almer, "Structural aspects of elastic deformation of a metallic glass," Physical Review BCondensed Matter and Materials Physics, vol. 73, no. 6, Article ID 064204, 8 pages, 2006.

[16] J. Ding, Y. Q. Cheng, and E. Ma, "Correlating local structure with inhomogeneous elastic deformation in a metallic glass," Applied Physics Letters, vol. 101, no. 12, Article ID 121917, 2012.

[17] M. L. Falk, J. S. Langer, and L. Pechenik, "Thermal effects in the shear-transformation-zone theory of amorphous plasticity: comparisons to metallic glass data," Physical Review EStatistical, Nonlinear, and Soft Matter Physics, vol. 70, no. 1, Article ID 011507, 11 pages, 2004.

[18] V. V. Bulatov and A. S. Argon, "A stochastic model for continuum elasto-plastic behavior. III. Plasticity in ordered versus disordered solids," Modelling and Simulation in Materials Science and Engineering, vol. 2, no. 2, pp. 203-222, 1994.

[19] L. Qi, H. F. Zhang, Z. Q. Hu, and P. K. Liaw, "Molecular dynamic simulation studies of glass formation and atomic-level structures in Pd-Ni alloy," Physics Letters, A: General, Atomic and Solid State Physics, vol. 327, no. 5-6, pp. 506-511, 2004.

[20] C. A. Schuh and A. C. Lund, "Atomistic basis for the plastic yield criterion of metallic glass," Nature Materials, vol. 2, no. 7, pp. 449-452, 2003.

[21] H. L. Peng, M. Z. Li, and W. H. Wang, "Structural signature of plastic deformation in metallic glasses," Physical Review Letters, vol. 106, no. 13, Article ID 135503, 4 pages, 2011.

[22] D. Pan, A. Inoue, T. Sakurai, and M. W. Chen, "Experimental characterization of shear transformation zones for plastic flow of bulk metallic glasses," Proceedings of the National Academy of Sciences of the United States of America, vol. 105, no. 39, pp. 14769-14772, 2008.

[23] S. Plimpton, "Fast parallel algorithms for short-range molecular dynamics," Journal of Computational Physics, vol. 117, no. 1, pp. 1-19, 1995.

[24] M. I. Mendelev, D. J. Sordelet, and M. J. Kramer, "Using atomistic computer simulations to analyze $\mathrm{x}$-ray diffraction data from metallic glasses," Journal of Applied Physics, vol. 102, no. 4, Article ID 043501, 7 pages, 2007.

[25] A. J. Cao, Y. Q. Cheng, and E. Ma, "Structural processes that initiate shear localization in metallic glass," Acta Materialia, vol. 57, no. 17, pp. 5146-5155, 2009.
[26] Y. Zhang, N. Mattern, and J. Eckert, "Effect of uniaxial loading on the structural anisotropy and the dynamics of atoms of $\mathrm{Cu}_{50} \mathrm{Zr}_{50}$ metallic glasses within the elastic regime studied by molecular dynamics simulation," Acta Materialia, vol. 59, no. 11, pp. 4303-4313, 2011.

[27] F. Shimizu, S. Ogata, and J. Li, "Theory of shear banding in metallic glasses and molecular dynamics calculations," Materials Transactions, vol. 48, no. 11, pp. 2923-2927, 2007.

[28] S. D. Feng, L. Qi, G. Li, W. Zhao, and R. P. Liu, "Effects of preintroduced shear origin zones on mechanical property of $\mathrm{ZrCu}$ metallic glass," Journal of Non-Crystalline Solids, vol. 373-374, pp. 1-4, 2013.

[29] Y. F. Shi and M. L. Falk, "Atomic-scale simulations of strain localization in three-dimensional model amorphous solids," Physical Review B-Condensed Matter and Materials Physics, vol. 73, no. 21, Article ID 214201, 10 pages, 2006.

[30] W. Brostow, M. Chybicki, R. Laskowski, and J. Rybicki, "Voronoi polyhedra and Delaunay simplexes in the structural analysis of molecular-dynamics-simulated materials," Physical Review B-Condensed Matter and Materials Physics, vol. 57, no. 21, pp. 13448-13458, 1998.

[31] Y. Q. Cheng, A. J. Cao, H. W. Sheng, and E. Ma, "Local order influences initiation of plastic flow in metallic glass: effects of alloy composition and sample cooling history," Acta Materialia, vol. 56, no. 18, pp. 5263-5275, 2008.

[32] C. E. Lekka, A. Ibenskas, A. R. Yavari, and G. A. Evangelakis, "Tensile deformation accommodation in microscopic metallic glasses via subnanocluster reconstructions," Applied Physics Letters, vol. 91, no. 21, Article ID 214103, 3 pages, 2007. 

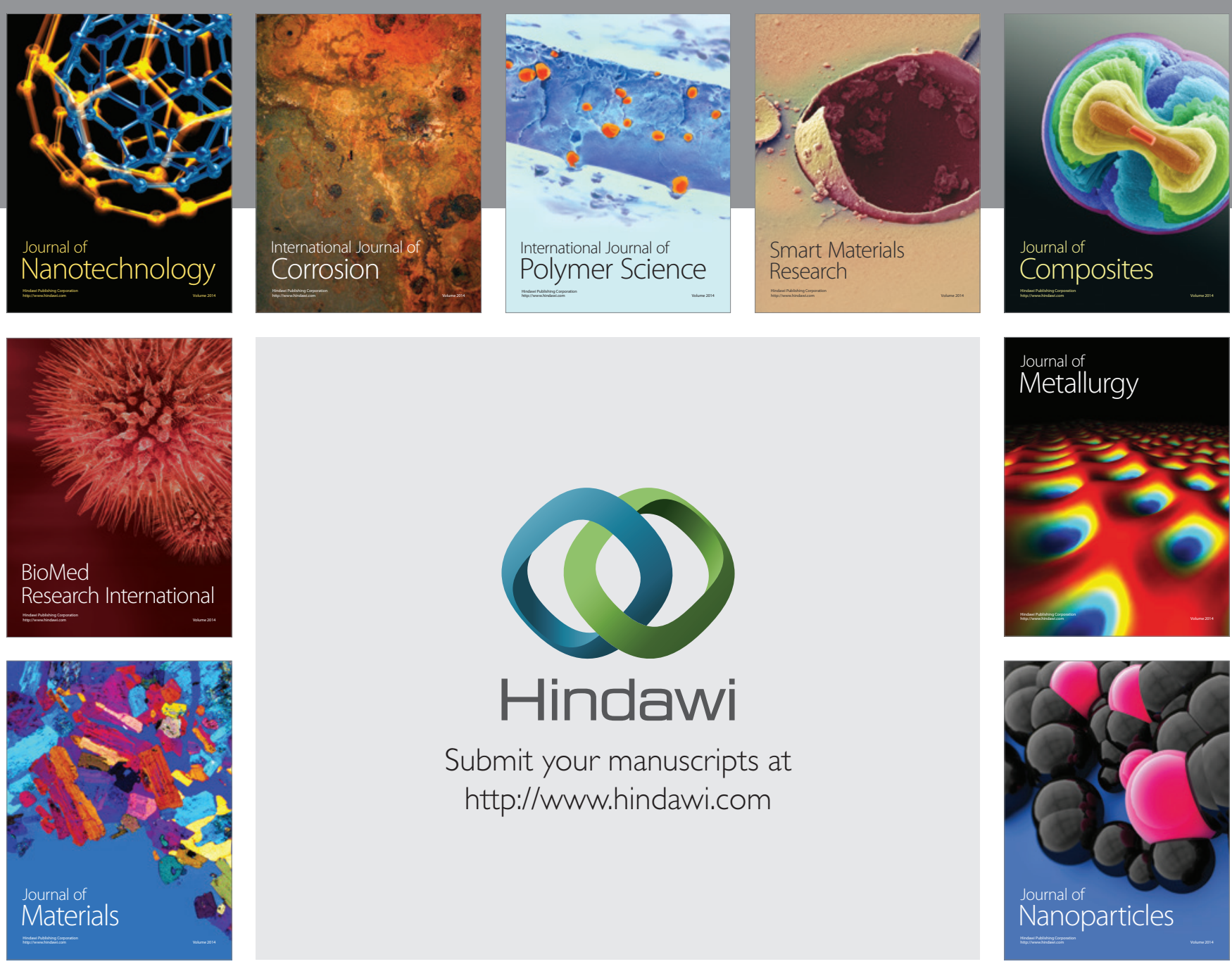

Submit your manuscripts at http://www.hindawi.com
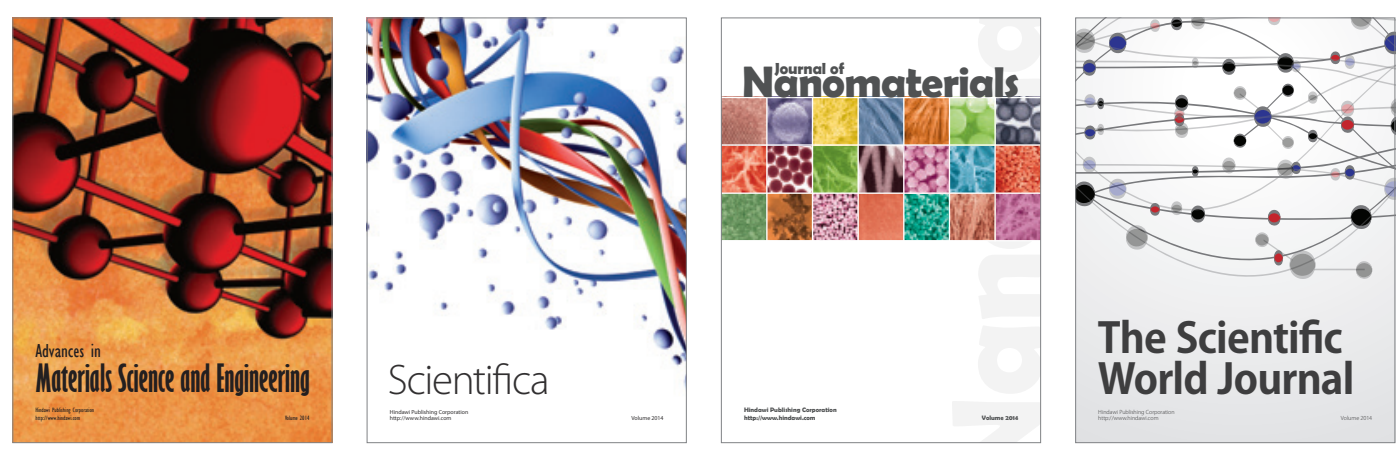

\section{The Scientific World Journal}
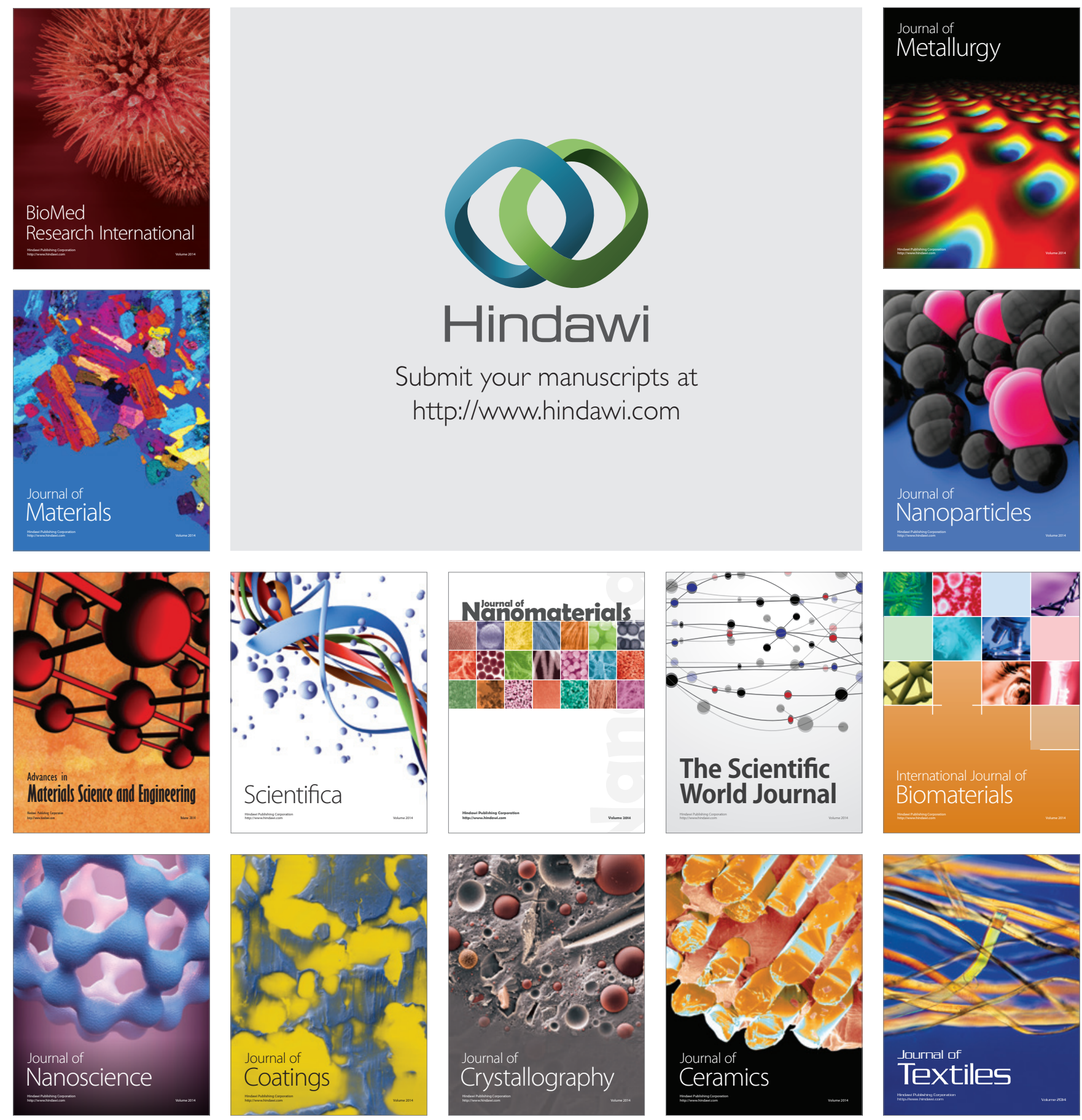